\title{
A cooperação intermunicipal no Brasil: 0 caso dos consórcios de resíduos sólidos
}

\author{
Dituzaya Panguila da Silva ${ }^{1}$ \\ Hugo Consciência Silvestre ${ }^{2}$ \\ Alfa Aliu Embalo ${ }^{3}$ \\ 1 Instituto Universitário de Lisboa-ISCTE, Lisboa - Portugal \\ 2 Universidade da Integração Internacional da Lusofonia Afro-brasileira / Núcleo de Políticas \& Administração Pública, Redenção / \\ CE - Brasil \\ ${ }^{3}$ Instituto Superior de Educação e Ciências de Lisboa-ISEC, Lisboa - Portugal
}

\begin{abstract}
Esta pesquisa tem como principal objetivo descrever a relação entre a utilização dos consórcios com os custos da cooperação pública e de seu financiamento por parte dos municípios. Pela utilização do desenho de pesquisa de caso múltiplo, selecionaram-se três consórcios a atuar no setor dos resíduos sólidos no Brasil, compreendendo o intervalo de anos entre 2013 e 2017. Em geral, é possível concluir que os custos com os consórcios e dos municípios aumentaram durante o período. Pela estimativa de parâmetros, estima-se que os consórcios possibilitam a redução desses custos. Conclui-se ainda que governos locais de menor dimensão, em número de habitantes e área servida, são mais propensos a potenciar os efeitos da cooperação pública. Implicações teóricas e práticas serão apresentadas e discutidas ao longo do texto.
\end{abstract}

Palavras-chave: cooperação pública; redes ou networks; custos; resíduos sólidos; Brasil.

\section{Cooperación intermunicipal en Brasil: el caso de los consorcios de residuos sólidos}

En este artículo pretendemos describir la relación entre la cooperación intermunicipal con los costos organizacionales y financieros. Sobre la base de un diseño de investigación de casos múltiples, se seleccionaron tres consorcios operantes en el sector brasileño de residuos sólidos en el período 2013-2017. Los principales hallazgos de este estudio muestran que tanto los costos organizacionales como financieros aumentaron durante el período. Sin embargo, y a partir del cálculo de un estimador de parámetros, es probable que dichos costos se reduzcan en el futuro cercano. También se puede concluir que los gobiernos locales más pequeños (ya sea por población o por $\mathrm{km} 2$ ) tienen más probabilidades de aumentar los efectos de la cooperación pública. Las implicaciones teóricas y prácticas de los hallazgos de este estudio se discuten a lo largo del artículo.

Palabras clave: cooperación pública; redes; costos; residuos sólidos; Brasil.

\section{Inter-municipal cooperation in Brazil: the case of solid waste consortia}

This article aims to describe the engagement of local governments in intermunicipal cooperation and the costs of these arrangements for municipalities. Based on a multiple-case research design, three consortia operating in the Brazilian solid waste sector between 2013 and 2017 were selected and examined. The main findings show that the costs of the intermunicipal cooperation and the costs related to the participation of the municipality in these arrangements increased in the period. However, a parameter estimator calculation shows that such costs are likely to be reduced in the near future. It can also be concluded that smaller local governments (either by population or by $\mathrm{km} 2$ ) are more likely to benefit from intermunicipal cooperation. The study discusses both theoretical and practical implications.

Keywords: public cooperation; networks; costs; solid waste; Brazil. 


\section{AGRADECIMENTOS}

Agradecemos ao CNPq pelo financiamento desta pesquisa, processo no 426771/2018-4 CNPq.

\section{INTRODUÇÃO}

A utilização dos mercados para a prestação de serviços públicos surgiu como alternativa à hierarquia ou ao modelo burocrático que vigorava até a década de 1980. A hierarquia era tida como ineficiente na utilização dos recursos humanos, técnicos, financeiros e materiais porque seu principal objetivo passava pelo cumprimento das regras escritas. O excesso de formalização para o cumprimento das tarefas não possibilitava equacionar novos modos de elevar a eficiência da administração pública tradicional (Osborne, 2006, 2010).

Dessa forma, despontou a nova gestão pública, que defende a participação das organizações do setor privado na prestação de serviços públicos. A participação dos privados ocorreria pela utilização dos mercados e, ao mesmo tempo, pela competição entre operadores (Pollitt \& Bouckaert, 2017). A competição deveria ser promovida para que a eficiência na prestação dos serviços pudesse melhorar. Ao operando usualmente em mercados competitivos, assume-se que as organizações do setor privado desenvolvem suas técnicas gerenciais. O desenvolvimento dessas técnicas lhes permite aumentar a eficiência e garantir a própria sobrevivência. Por essas razões, deveriam os privados participar da prestação de serviços tendo em vista a redução dos custos e a melhoria da eficiência.

À época, e a par da utilização da competição por meio do uso dos mercados, a descentralização foi amplamente desenvolvida (Pollitt, 1990), tendo como objetivo garantir maior autonomia política, administrativa e financeira para o nível mais básico de prestação dos serviços públicos: governos locais ou municípios. O modelo foi considerado atraente e acreditava-se que tornaria a prestação dos serviços públicos mais eficientes, econômicos e de maior efetividade, possibilitando a diminuição dos custos aos seus financiadores (Denhardth \& Denhardth, 2000).

Em que pese o fato de ter sido difundida a participação das organizações do setor privado na prestação de serviços públicos, não ficou comprovado que seu envolvimento tenha levado à redução dos custos dos serviços (Bel \& Fageda, 2015). Assim, tornou-se necessário repensar novos modos de prestação dos serviços públicos, sobretudo após a crise financeira mundial de 2008 e a crise nacional de 2015.

A cooperação pública vem sendo equacionada como um possível arranjo para diminuir os custos de prestação de serviços públicos ao mesmo tempo que eleva sua quantidade e qualidade. A cooperação pública não é um fenômeno novo, porém é assumida como ferramenta para a reforma do setor público dentro do modelo da nova governança pública (Torfing \& Sorensen, 2014). O modelo se baseia em duas premissas básicas: a participação dos vários atores sociais na coconstrução das políticas públicas e sua coimplementação (Osborne, 2006, 2010). A participação dos vários atores sociais na coconstrução de políticas públicas, em especial a participação cidadã, eleva a democratização e, por consequência, a legitimidade política da coisa pública (Peters, 2004). Trata-se, portanto, de uma nova forma de governar (Christesen, 2012), alicerçada nos princípios de responsabilidade, integridade e transparência (Santos \& Giovanella, 2014).

A cooperação se assenta em redes ou networks para a prestação de serviços envolvendo múltiplos atores (Rhodes, 2007) - entre eles, sobressai a cooperação intermunicipal. Em teoria, esse arranjo tende 
a promover a redução dos custos organizacionais e operacionais, bem como o investimento realizado pelos principais: os governos locais. Mas essas redes funcionam realmente? (Provan \& Milward, 1999). Segundo Bel, Fageda, e Warner (2010), a cooperação intermunicipal mostra potencial para a redução dos custos na prestação de serviços públicos. Porém, Sabourin, Massardier, e Sotomayor (2016) revelam que existe o risco de apenas se utilizar a retórica sem que exista uma efetiva implementação de políticas públicas e melhoria da ação coletiva. Em explicação, Caldas e Moreira (2013) defendem que a maior preocupação dos atores poderá se centrar nos processos, em vez de conteúdos e regras, não possibilitando maior efetividade dessa ação. Já Massardier e Sabourin (2013) apontam para a existência de múltiplos objetivos da ação coletiva, sobretudo quando perante a governança local e o desenvolvimento regional no contexto da América Latina. Pelos argumentos ora expostos, o conhecimento sobre se a cooperação em rede de fato funciona demanda estudos que o esclareçam e comprovem.

Em função da problemática de pesquisa que agora se coloca, pretende-se descrever a relação entre a utilização da cooperação intermunicipal com os custos da cooperação e de seu financiamento. Para a prossecução, selecionou-se o setor de resíduos sólidos no Brasil. A maioria dos estudos realizados versa sobre a realidade ocidental, carecendo de evidências empíricas contextuais várias, como é o caso da América Latina. Destaca-se a realidade nacional, cuja cooperação pública tem longa tradição. Estudos têm sido realizados ao caso nacional, mas a maioria trata do setor da saúde.

Não obstante o setor, verifica-se que os estudos nacionais seguem a abordagem adotada pelos pesquisadores da América do Norte, que privilegia o conhecimento dos fatores que levam à cooperação pública (Abrúcio, Filippim, \& Dieguez, 2013; Abrúcio, Sano, \& Sydow, 2010; Matos \& Dias, 2011, entre outros). Em consideração, essa tendência decorre da questão federativa por ambas partilhada, ou seja, a existência de três níveis de governo. As relações entre entes federativos se baseiam no financiamento e na melhoria dos serviços públicos de espectro intergovernamental ou multinível. No caso particular do Brasil, justifica-se ainda essa opção pela elevada heterogeneidade dos seus municípios, o que implica as capacidades estatais de cada uma das unidades. Neste estudo, privilegia-se a abordagem adotada pelos pesquisadores da Europa, que se tem centrado nos fatores que influenciam os custos organizacionais e operacionais para a prestação de serviços públicos por meio da cooperação intermunicipal (Bel \& Warner, 2015). Crê-se que esta seja outra inovação do estudo que agora se propõe.

Pela estratégia empírica, adotou-se o desenho de estudo de caso múltiplo (Gerring, 2004) com o objetivo de analisar e comparar os casos selecionados por meio da análise de congruência (Blatter \& Blume, 2008). A seleção dos consórcios se deu pela técnica de amostragem intencional (Jooste \& Scott, 2012). A seleção possibilitou a inclusão de 3 consórcios a atuar no setor dos resíduos sólidos e com sede no Rio Grande do Sul, em Santa Catarina e em Minas Gerais. Para o tratamento dos dados, fez-se uso da análise univariada de variância e, dentro dessa, da regressão por quadrados mínimos ponderados e do teste de efeitos entre sujeitos.

Após a Introdução, detalha-se a governança em rede e seus pressupostos teóricos. Em seguida, apresenta-se a cooperação intermunicipal como arranjo para a prestação de serviços públicos, incluindo a análise dos estudos já realizados. Depois de apresentada a estratégia empírica, apresentam-se os resultados e sua discussão. O trabalho finda com a conclusão. 


\section{A GOVERNANÇA EM REDE}

\subsection{Enquadramento}

A governança pública trata do direcionamento da sociedade em que diferentes atores, governamentais e não governamentais, participam na prossecução do bem-estar social (Klijn, 2008; Santos \& Giovanella, 2014). A governança pública tem como objetivo estreitar as relações de cooperação entre governo e sociedade civil, bem como com as organizações do setor público e privado. Justifica-se essa abordagem pela inclusão de múltiplos atores para a prestação de serviços públicos, que eleva a capacidade governamental de providenciar os serviços necessários às populações (Bevir \& Rhodes, 2001).

Até a década de 1980, a hierarquia ou o modelo burocrático era preferencialmente utilizado para a prestação de serviços públicos. A utilização desse modelo se justificava pela supervisão direta que atores políticos e administradores públicos, em resultados da definição de autoridade, exerciam sobre a execução dos processos administrativos. A execução das tarefas estava bem definida por regras escritas (Osborne, 2006, 2010). Essa formalização possibilitava o controle sobre o cumprimento das atividades, razão pela qual o modelo perdurou. As crises petrolíferas vivenciadas exigiram, contudo, repensar o arranjo organizacional e seu financiamento (Pollitt, 1990).

As crises petrolíferas tiveram como consequência o desacelerar da atividade econômica. Com seu abrandamento, as trocas comerciais diminuíram consideravelmente, encurtando os impostos coletados pelos governos. O problema surge porque a demanda por serviços públicos aumentou em virtude do menor poder de compra da população. Além disso, a necessidade de reforçar a assistência social veio a pressionar as já deficitárias contas públicas primárias (Pollitt \& Bouckaert, 2017). Adiciona-se às condições econômicas e financeiras vivenciadas as críticas realizadas pelos neoliberais à utilização do modelo de hierarquia. Para eles, o governo seria o responsável pelas políticas públicas, mas não necessariamente pela prestação de serviços efetivada por meio da burocracia, que era ainda tida como ineficiente na utilização dos recursos disponíveis. Portanto, a prestação de serviços deveria ocorrer pela utilização dos mercados, pela qual a eficiência organizacional e operacional seria melhorada, ao mesmo tempo que possibilitaria a redução do pagamento de impostos por parte dos cidadãos (Silvestre, 2010, 2019).

Pelas razões descritas, despontou o modelo da nova gestão pública (Hood, 1991). Como já referenciado, esse modelo se apoiava na competição entre operadores para que fosse possível a redução dos custos da prestação de serviços públicos. Preferencialmente, as organizações do setor privado é que deveriam entregar esses serviços aos usuários. Pela competição entre elas, e em teoria, os custos operacionais tenderiam a diminuir.

A par do aumento da competição, a descentralização administrativa foi amplamente incentivada, razão pela qual os governos locais ou municípios assumiram maiores responsabilidades na provisão e na prestação de serviços públicos. Justifica-se essa opção pelo conhecimento profundo que os atores políticos e administrativos locais têm sobre as reais necessidades da população que servem em resultado das relações de proximidade que mantêm. Mesmo existindo esse movimento de descentralização, a participação das organizações do setor privado na prestação de serviços públicos deveria ser privilegiada (Pollitt \& Bouckaert, 2017).

Embora tenha sido suportada como estratégia de reforma institucional e administrativa, a participação dos privados na prestação de serviços não comprovou que os custos fossem de fato 
reduzidos (Bel \& Warner, 2015). Por essa motivação, vem-se discutindo a cooperação pública por meio de redes ou networks, como arranjo possível para aumentar a eficiência dos serviços públicos e reduzir seus custos.

\subsection{A nova governança pública}

Ainda na década de 1980, as instâncias governamentais iniciaram o estreitamento de relações com outros atores sociais, em especial com organizações da sociedade civil e voluntariado do terceiro setor, entre outros. Segundo Rhodes (2000), uma das principais motivações para essa proximidade se deveu à necessidade de repensar a concepção das políticas públicas e sua execução. Até então, a decisão sobre os programas a serem implementados, assim como sua execução, era definida centralmente, sem uma ampla participação dos vários atores.

A participação dos demais atores na definição das políticas públicas e na sua execução tem sido promovida pela nova governança pública, termo cunhado por Osborne, em 2006, em artigo publicado na Public Management Review. Esse modelo se pauta na ideia de que os atores sociais devem apelar para a coconstrução ou a participação dos vários atores sociais na definição de políticas públicas. Já para sua execução, a coimplementação deve ser privilegiada, sobretudo por meio do conhecimento especializado dos usuários dos serviços (Osborne, 2006, 2010). Crê-se que, pela participação dos vários atores em ambas as fases, as políticas a serem executadas levam a uma melhoria das condições de vida dos usuários, que podem auxiliar na sua implementação com disponibilidade e conhecimentos - aquilo que se tem chamado de coprodução (Oström, 1996).

A governança pública se associa, desse modo, ao aumento dos efeitos dos programas governamentais mediante a interação de atores governamentais e não governamentais, a participação cidadã, a utilização de redes para prestação de serviços públicos, a mudança das técnicas de gestão da coisa pública, o aumento da transparência, a diminuição dos gastos públicos e o aumento da eficiência na utilização dos recursos (Bevir \& Rhoes, 2001; Hans \& Klijn, 2008; Klijn, 2008). Trata-se de uma nova abordagem pela qual as políticas públicas são negociadas com outros atores. Procura-se, com essa negociação, o desenvolvimento da capacidade estatal na resposta aos anseios dos usuários dos serviços (Abrúcio, 1997).

Segundo Provan e Milward (1999), a governança pública poderá ser concretizada por meio de três níveis de cooperação. O primeiro é o nível da comunidade, envolvendo a relação entre o principal, o governo, os atores interessados na prestação e os beneficiários dos serviços. Ele se centra na definição das políticas públicas a serem idealizadas. O segundo é o nível de redes ou networks, pelo qual se alinham as relações entre o principal e os agentes executores dos serviços, tendo em vista a implementação da política previamente definida. Por fim, há o nível da organização na sua relação de participação com os usuários dos serviços. Por ser central ao estudo, passamos em revista o nível de redes ou networks.

\subsection{A cooperação em rede ou networks para a prestação de serviços públicos}

As redes ou networks foram desenvolvidas pelo fenômeno da descentralização ocorrido ainda durante a vigência da nova gestão pública. Tal fato despontou no lançamento do programa Os próximos passos (The Next Steps), em 1988 (Silvestre, 2010). O histórico desenvolvimento do governo britânico após a 
Segunda Guerra Mundial exigiu a adoção de uma agenda de reforma administrativa e institucional. A descentralização fez com que as redes ou networks fossem prioritárias para a prestação de serviços públicos em nível dos governos locais. Essas redes servem de contraditório aos valores da verticalidade do poder central existentes à época (Bevir \& Rhodes, 2001; Osborne, 2006).

Como conceito, as redes compreendem a cooperação voluntária e a coordenação entre duas ou mais unidades em que as principais componentes poderão não estar inclusas numa única estrutura (Feiock, 2007; O'toole \& Meier, 2004). As unidades envolvidas poderão incluir os governos - federal, estadual ou municipal - na relação com organizações do setor privado e com associações sem fins lucrativos ou voluntárias, as quais têm como objetivo prioritário a melhoria do processo de tomada de decisão pelo desenvolvimento do processo e dos efeitos das políticas. A junção de organizações para prestação de serviços públicos é movida pelo espírito mútuo, diplomático e de confiança entre os parceiros dentro da rede (Rhodes, 2007). As redes ganham papel funcional de sistemas facilitadores na implementação de ações governamentais dentro e fora dos departamentos governamentais, em antecipação a limites e oportunidades gerenciais (Klijn, Koppenjan, \& Temeer, 1995).

Klijn, Koppenjan, e Termeer (1995) afirmam que a formação em rede, aquelas que se formam e incluem entidades interdependentes, apresenta como grande vantagem o aproveitamento dos recursos políticos, técnicos e administrativos dos cooperantes. A cooperação é voluntária, o que requer uma relação pautada por reciprocidade, respeito mútuo, comprometimento e honestidade. Já os fatores que afetam a qualidade, a quantidade da prestação do serviço e os custos incorridos deles são mantidos como estratégias para que governos locais prestem os serviços na forma desejável sem necessariamente desqualificá-los.

Para a execução da política por meio de redes, vários arranjos poderão ser utilizados, como os acordos de cooperação, cooperação intermunicipal, entre outros (Agranoff, 2014). Em se tratando da cooperação pública entre municípios, é comum a utilização da cooperação intermunicipal (Bel, Dijkgraaf, Fageda, \& Gradus, 2010). Nesse particular, tais redes de governança são caracterizadas por uma relação horizontal (Klijn, 2008; Hulst \& Montfort, 2012). Pelo esforço conjunto, procura-se mitigar os problemas sociais, ambientais, políticos e econômicos localizados (Cruz, 2001; Feiock, 2007). As unidades cooperantes assumem que cada um dos atores envolvidos é elemento capital para a concretização dos objetivos comuns. Esses objetivos se suportam na legitimidade do sistema político, na responsabilidade, na transparência e na interdependência das partes.

Entre as motivações para a cooperação pública em nível municipal, desponta a possibilidade de diminuição de custos na prestação dos serviços que estão obrigados, por lei, a prover (Leroux \& Carr, 2007). A redução dos custos poderá ser conseguida pelo aproveitamento das economias de escala, que se firmam na redução dos custos médios do serviço que poderão advir do maior número de habitantes e área (Feiock, 2007; Hefetz \& Warner, 2011).

Outra das motivações aponta para a diminuição dos oportunismos das empresas privadas quando perante a contratação pública (Feiock, 2007; Warner, 2011). Custos de contratação e monitoramento dos contratos - os custos de transação (Williamson, 1999) - se associam, em grande parte, à contratação tradicional pela qual o agente público contrata o privado para a prestação de determinado serviço (Shrestha \& Feiock, 2011; Bel, Fageda, \& Mur, 2012). Essa explicação é baseada na contratação pública, pela qual os interesses privados poderão colidir com os interesses públicos. Daí que os custos de transação elevem os administrativos, ou seja, pela possibilidade de contratos incompletos, mas também de monitoramento e controle (Williamson, 1999; Bel, Fageda, \& Mur, 2012). 


\subsection{A institucionalização da cooperação no contexto brasileiro}

A Constituição Federal de 1988 trouxe, entre outras coisas, a descentralização e o empoderamento dos municípios no Brasil no que tange à autonomia política, financeira e administrativa (Mello, 1997). Embora prevista desde a década de 1930, a cooperação como movimento de reforma tem seu maior avanço durante a década de 1980 (Abrúcio, Sano, \& Sydow, 2010). No artigo 23 da Constituição Federal, está prevista a existência de acordos de cooperação entre os governos federal, estadual e local (Linhares et al., 2017). Esses acordos poderão ser efetivados pelas regiões metropolitanas, pelos consórcios intermunicipais e pelos planos de desenvolvimento regionalizados (Spink, 2005).

No que respeita aos consórcios intermunicipais, os serviços são mantidos por uma administração conjunta e tradicionalmente horizontalizada. Amparados pela lei $\mathrm{n}^{\circ} 11.107$, de 6 de abril de 2005 (Machado \& Andrade, 2014), e pelo decreto $n^{\circ} 6.017$, de 2007, os consórcios são regidos pelo direito público porquanto o financiamento ou a manutenção destes depende das finanças públicas municipais.

A cooperação pública se tornou um tema muito importante nacionalmente em resultado das necessidades de alargamento de prestação de serviços públicos e das constantes dificuldades para garantir seu financiamento (Abrúcio, Filippim, \& Dieguez, 2013). Desde o movimento de descentralização se vem questionando a capacidade administrativa dos municípios, a fim de desenharem os serviços de que as populações necessitam (Laczynski \& Teixeira, 2012). Na realidade, os governos locais nacionais $(n=5,570)$ apresentam, em sua maioria, uma baixa capacidade financeira para manter os serviços, em resultado da pequena dimensão (Lima \& Silveira, 2018).

Como defende Cunha (2004), a possibilidade de aproveitamento das economias de escala por parte dos governos locais nacionais só é possível pela cooperação pública. Estratégia essa que se torna mais importante para os municípios com menos de 20 mil habitantes, os quais compõem $70 \%$ do total dos governos locais nacionais (Henrichs \& Meza, 2017). Em 2018, foram identificados 3977 acordos de cooperação envolvendo, na maioria, governos locais. Os setores de saúde, resíduos sólidos e ambientais são aqueles que apresentam o maior número de casos de cooperação, em que os municípios com menos de 20 mil habitantes apresentam maior participação - em torno de $72 \%$ (Silvestre et al., 2019).

A utilização da cooperação intermunicipal no Brasil, por meio dos consórcios, apresenta as mesmas motivações e preocupações em comparação com casos internacionais. Resta, portanto, perceber os estudos realizados e os resultados constatados.

\subsection{Estudos realizados e principais resultados}

Os estudos realizados em torno da cooperação intermunicipal seguem duas abordagens distintas (Bel \& Warner, 2015). A primeira vem sendo desenvolvida na América do Norte, em especial nos Estados Unidos. A preocupação maior dessas pesquisas está no conhecimento das motivações e dos fatores que levam à cooperação pública (Feiock, 2007).

Nessas pesquisas, conclui-se que a cooperação intermunicipal é influenciada pela necessidade de atingimento das economias de escala. Confrontados com dificuldades financeiras, as quais impedem que os governos locais possam atender às demandas das populações, a cooperação intermunicipal surge como alternativa ao aproveitamento e ao compartilhamento dos recursos entre essas unidades. Conclui-se que, pela criação de sinergias inter-organizacionais e pelo favorecimento no aproveitamento das economias de escala, a cooperação intermunicipal tem sido benéfica para a prestação de serviços 
públicos (Feiock, 2007; Bel, Dijkgraaf, Fageda, \& Gradus, 2010; Hefetz \& Warner, 2011; Hulst \& Montfort, 2012).

Essa linha de pesquisa tem sido seguida no Brasil, e tal como referem Abrúcio, Filippim, e Dieguez (2013), a vontade política e o conhecimento dos fatores que contribuem para o engajamento nos consórcios têm sido privilegiados. Os estudos realizados à cooperação consorciada vêm sendo centrados no setor da saúde e de recursos hídricos (Cruz, 2001; Silveira \& Phillipi, 2008; Mello \& Lago-Peña, 2013).

Por sua vez, os acadêmicos do continente europeu têm como interesse maior o conhecimento dos custos e dos efeitos da cooperação pública, e não tanto no conhecimento sobre os fatores que levam ou não à cooperação. Nesse particular, Allers e Greef (2018) não identificam qualquer redução nos gastos quando os municípios holandeses cooperam. Já Niaounakis e Blank (2017) concluíram pela redução dos custos das unidades cooperantes. Segundo os autores, a redução dos custos teve origem no aproveitamento das economias de escala. Na Espanha, Bel, Fageda, e Mur (2012), além de ZafraGómez, Prior, Díaz, e López-Hernández (2013), verificaram que a cooperação intermunicipal leva a uma diminuição dos custos. Na França, Frère, Leprince, e Paty (2014) não identificaram qualquer relação entre os níveis de despesa e a cooperação intermunicipal.

No Brasil, a academia tem sido frutífera nos estudos sobre o setor dos resíduos sólidos e inúmeros trabalhos vêm sendo realizados. Nauro (2003) reporta que a gestão de resíduos sólidos é benéfica para os pequenos municípios quando engajados em ações consorciadas. Comparativamente, a eficiência é maior e os custos são menores em relação aos municípios que prestam esse serviço de forma isolada. Por sua vez, baseado em estudo de caso, Calderan (2013) conclui que a cooperação consorciada melhora a capacidade administrativa para a prestação de serviços, entre outras benfeitorias. Ferreira e Juca (2017) suportam a ideia de que essa capacidade consorciada poderá ser elevada pelo desenvolvimento de tecnologias mais eficazes.

Como referenciado, há estudos sobre o setor dos resíduos sólidos no Brasil. O presente artigo se centra, porém, nos custos operacionais e de financiamento dos próprios consórcios para a prestação dos serviços de resíduos sólidos.

\section{ESTRATÉGIA EMPÍRICA}

Pela revisão da literatura, a cooperação pública vem sendo efetivada com o objetivo de diminuir os custos das organizações do setor público ao mesmo tempo que se potencializa a quantidade e a qualidade na prestação dos serviços. Nesse particular, tem despontado a cooperação entre municípios, assumindo-se como uma alternativa à utilização da hierarquia e dos mercados (Silvestre, Marques, \& Gomes, 2018). Desse modo, e com influência da abordagem da nova governança pública, as redes de cooperação entre organizações do setor público têm sido privilegiadas (Osborne, 2006, 2010). A cooperação pelas redes e como já referido não é algo novo. A novidade surge pela sistematização na utilização destas para a realização da reforma institucional e administrativa (Torfing \& Sorensen, 2014).

Por essas razões, o objetivo neste estudo passa por descrever a relação entre a utilização da cooperação intermunicipal com os custos da cooperação e de seu financiamento. Essa descrição se traduz, necessariamente, pela identificação de quais variáveis independentes e contextuais melhor se relacionam estatisticamente com as variáveis dependentes. Num segundo momento e pela estimativa de parâmetros, tem-se como objetivo relacionar o comportamento das variáveis do modelo. 


\subsection{Desenho de pesquisa e unidade de análise}

Pela necessidade de conhecer a utilização dos consórcios na sua relação com os custos para os governos locais, selecionou-se o desenho de pesquisa de tipo estudo de caso múltiplo (Blaikie \& Priest, 2019). O desenho de pesquisa selecionado teve como principal quesito o relacionamento dos casos em análise (Gerring, 2004). Para que esses casos possam ser analisados e comparados, utiliza-se a análise de congruência. Segundo Blatter e Blume (2008), bem como Stewart (2012), a comparação dos casos torna possível a identificação, a explicação e o entendimento da relação de causa e efeito do fenômeno social num contraponto com a revisão da literatura.

A seleção dos consórcios se deu pela técnica de amostragem intencional (Jooste \& Scott, 2012). Num primeiro momento, o setor dos consórcios dos resíduos sólidos foi escolhido. Como referem Abrúcio, Filippim, e Dieguez (2013), assim como Abrúcio, Sano, e Sydow (2010), estudos nacionais sob a temática existem, mas a maioria analisa diferentes setores. Assim, a opção pelo setor de resíduos sólidos é uma inovação desta pesquisa. Ainda nesse quesito e numa perspetiva comparativa, mostra-se possível a comparação dos resultados deste estudo com outros já realizados nacional e internacionalmente.

As pesquisas internacionais apontam para a existência de uma relação estatística significativa dos fatores contextuais com os custos do serviço (Bel \& Warner, 2015). Entre esses fatores contextuais, despontam a área servida e o número de habitantes. Por essa razão, a seleção dos consórcios foi idealizada tendo em consideração as diferenças entre esses fatores. Assim, selecionou-se os seguintes consórcios: Cigres, Cirsures e Ecotre, que compreendem $(n=) 41$ unidades de análise, sendo que o primeiro envolve a cooperação entre 31 governos locais; o segundo, entre 7 governos locais; e o terceiro, entre 3 governos locais, respectivamente.

\section{TABELA 1 CARATERIZAÇÃO DOS CONSÓRCIOS}

\begin{tabular}{|c|c|c|c|c|}
\hline Consórcio & Serviços & $\begin{array}{l}\mathrm{N}^{\circ} \text { de } \\
\text { municípios }\end{array}$ & Habi., média & $\begin{array}{l}\text { Área } \\
\left(\mathrm{km}^{2}\right) \text {, } \\
\text { média }\end{array}$ \\
\hline Cigres (RS) & $\begin{array}{l}\text { Coleta seletiva/Reciclagem/Aterro } \\
\text { Sanitário }\end{array}$ & 31 & $5.924,87$ & 161,19 \\
\hline Cirsures (SC) & $\begin{array}{l}\text { Coleta seletiva/Reciclagem/Aterro } \\
\text { sanitário }\end{array}$ & 7 & 15.823 & 235 \\
\hline Ecotre (MG) & $\begin{array}{l}\text { Coleta seletiva/ Reciclagem/ } \\
\text { Compostagem/Aterro } \\
\text { Sanitário }\end{array}$ & 3 & 40.049 & 311 \\
\hline
\end{tabular}

Fonte: Elaborada pelos autores com base nos dados coletados.

A par da escolha dos consórcios, discute-se o momento de análise. Essa reflexão foi feita em torno de dois eixos. A existência dos dados foi o primeiro desses eixos, ou seja, foi necessário assegurar que a maturidade dos próprios consórcios permitisse a disponibilidade de informação. Por exemplo, o 
governo local de Siderópolis integrou a cooperação pública para a coleta e o tratamento de resíduos sólidos pela Cirsures a partir de 2013. Tornou-se, portanto, necessário assegurar certa estabilidade ao número de integrantes dos consórcios, tendo em consideração a robustez dos resultados estatísticos.

No segundo eixo, tornou-se crucial que os dados coletados pudessem captar a realidade econômica nacional recente. Até 2014, o Brasil vivenciou um aumento do Produto Interno Bruto (PIB) que perdurava fazia mais de uma década. Tal evolução veio a ser interrompida no início de 2015, com a crise econômica que o país vivenciou. Como consequência, o PIB diminuiu, colocando as contas públicas sob pressão. Em resultado, foi necessário diminuir as transferências para os municípios, amplamente dependentes do Fundo de Participação dos Municípios (FPM). Dependentes do fundo, há a possibilidade de diminuição dos investimentos para a prestação de serviços públicos em nível local. Essa constatação fez com que o período de recessão econômica fosse incluído (Lima \& Silveira, 2018). Ponderados os dois eixos, a coleta de dados sobre os custos dos consórcios selecionados compreendeu os anos de 2013 a 2017.

\subsection{Dados utilizados}

Tendo por base a escolha dos consórcios e o período em análise, coletaram-se os dados no Observatório dos Consórcios Públicos e do Federalismo (http://www.ocpf.org.br) e no Instituto Brasileiro de Geografia e Estatística (IBGE, 2018). A coleta dos dados seguiu a forma funcional adotada para a análise empírica:

$$
Y_{i t}=\alpha C O N_{i, t}+\lambda A N O_{i, t}+\beta P O P U_{i}+\chi A R E A_{i}+\mu_{i+} e_{i, t}
$$

Em que $Y_{i t}$

$$
\lambda \text { CToCons }_{i, t}=\beta_{\text {CCons }_{i, t}}+\chi \text { CGLocal }_{i, t}
$$

\begin{tabular}{|c|c|c|}
\hline Variável & Descrição & Justificativa \\
\hline \multicolumn{3}{|l|}{ Dependente } \\
\hline $\begin{array}{l}\text { Custo do consórcio } \\
\text { (CCons) }\end{array}$ & Custo total do consórcio, em $\mathrm{R} \$$ & $\begin{array}{l}\text { Allers e Greef (2018); Niaounakis e Blank } \\
\text { (2017); Bel, Fageda, e Mur (2012); Zafra- }\end{array}$ \\
\hline $\begin{array}{l}\text { Custo do governo local } \\
\text { (CGLocal) }\end{array}$ & Custo do governo local com o consórcio, em $\mathrm{R} \$$ & $\begin{array}{l}\text { Gómez et al. (2013); Frère, Leprince, e } \\
\text { Paty (2014); Bel e Warner (2015) }\end{array}$ \\
\hline $\begin{array}{l}\text { Custo total com } 0 \\
\text { consórcio (CToCons) }\end{array}$ & $\begin{array}{l}\text { Custos totais do consórcio, incluindo investimentos } \\
\text { realizados pelos governos locais, em } \mathrm{R} \$\end{array}$ & \\
\hline \multicolumn{3}{|l|}{ Independente } \\
\hline Consórcio (CONS) & $\begin{array}{l}\text { Variável nominal em que se identificam três consórcios: } \\
\text { Cigres, codificado com o número 1; Cirsures, codificado } \\
\text { com o número 2; e Ecotre, codificado com o número } 3\end{array}$ & Feiock (2007); Plata-Díaz et al. (2014) \\
\hline
\end{tabular}

\section{QUADRO 1 DESCRIÇÃO DE VARIÁVEIS}




\begin{tabular}{|c|c|c|}
\hline Variável & Descrição & Justificativa \\
\hline Ano (ANO) & $\begin{array}{l}\text { Variável nominal que identifica o período pré- } \\
\text { depressão econômica (de } 2013 \text { a 2015), codificado } \\
\text { por 1, e o período de depressão econômica ( } 2016 \text { e } \\
\text { 2017), codificado por } 2\end{array}$ & Lima e Silveira (2018) \\
\hline \multicolumn{3}{|l|}{ Contextual } \\
\hline População (POPU) & $\begin{array}{l}\text { Variável categórica em que os governos locais com } \\
\text { até } 20 \text { mil habitantes são codificados por 1, enquanto } \\
\text { os outros assumem o código } 2\end{array}$ & $\begin{array}{l}\text { Cunha (2004); Henrichs e Meza (2017); } \\
\text { Cruz e Marques (2012) }\end{array}$ \\
\hline Área (Área) & $\begin{array}{l}\text { Variável categórica em que os governos locais com } \\
\text { áreas de até } 157 \text { mil km² são codificados por } 1 \text {, } \\
\text { enquanto os outros assumem o código } 2\end{array}$ & $\begin{array}{l}\text { Lima e Silveira (2018); } \\
\text { Cruz e Marques (2012) }\end{array}$ \\
\hline
\end{tabular}

Fonte: Elaborado pelos autores com base nos dados coletados.

\section{TABELA 2 ESTATÍSTICA DESCRITIVA}

\begin{tabular}{lccccc} 
& N & Média & Desvio padrão & Mínimo & Máximo \\
Variável dependente & & & & & \\
CCons & 168 & .000001 & .9928745 & -2.6770 & 2.6770 \\
CGLocal & 114 & .000000 & .9902755 & -2.5446 & 2.5446 \\
CToCons & 180 & .000001 & .9932611 & -2.7000 & 2.7000 \\
Variável independente & & & & - & - \\
COM & 205 & 1.32 & .604 & - & - \\
ANO & 205 & 1.40 & .491 & - & - \\
Variável contextual & & & & - \\
POPU & 205 & 1.1463 & .35431 & - \\
AREA & 205 & 1.49 & .501 & - \\
\hline
\end{tabular}

Fonte: Elaborada pelos autores com base nos dados coletados.

\subsection{Variáveis dependentes}

$\lambda C$ ToCons ${ }_{i, t}$ O custo total do consórcio, medido em R $\$$. O custo total está dependente do custo do consórcio per se, isto é, dos custos operacionais da cooperação para a entrega de serviços de resíduos sólidos. É preciso, contudo, acrescer os custos dos municípios e por que estes financiam o serviço. A soma das duas variáveis se traduzirá numa perspectiva total de custos em que os próprios governos locais incorrem (Niaounakis \& Blank, 2017; Zafra-Gómez et al., 2013).

$\beta_{C C o n_{i, t}} \mathrm{O}$ custo com o consórcio, em $\mathrm{R} \$$. Os custos com o consórcio se assumem, extraordinariamente, como variável dependente. Essa opção objetiva a análise dos custos operacionais da cooperação pública, uma vez que se assume que essa cooperação poderá diminuir os custos da ação consorciada (Allers \& Greef, 2018). 


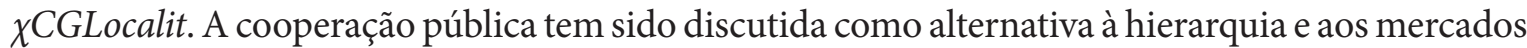
para a diminuição dos custos de prestação de serviços aos governos locais. Essa variável, também medida em R\$, reporta aos custos dos governos locais com o financiamento dos consórcios. Nessa motivação, assume-se como dependente no modelo (Bel \& Warner, 2015).

\subsection{Variáveis independentes}

$\alpha$ CONit. Cada consórcio foi considerado no modelo como variável independente - relembrando, os consórcios Cigres, Cirsures e Ecotre foram selecionados. Os consórcios se definem como uma rede ou network (Feiock, 2007), sendo expectável que as diferenças de população e área que os caracterizam possam ter efeito nos custos operacionais, nos custos dos governos locais e, por consequência, nos custos totais para a prestação de serviços de resíduos sólidos.

$\lambda A N O i t$. O ano aparece como variável independente, decorrente da crise financeira nacional, que leva a uma diminuição das transferências para os governos locais e do financiamento dos serviços públicos por eles prestados (Lima \& Silveira, 2018). Segundo Puye et al. (2018), a cooperação pública depende da capacidade financeira dos governos locais. Com a redução do FPM, o ano poderá ser uma variável com efeito nos custos operacionais.

\subsection{Variáveis contextuais}

Segundo Bel, Warner, e Hebdon (2012), as características contextuais em que as organizações do setor público operam poderão influenciar os custos da prestação dos serviços. Assim, despontam as seguintes variáveis de contextuais.

$\beta P O P U_{i, t^{*}}$ É crível que a existência de um maior número de residentes diminua o custo por unidade do serviço prestado. Segundo Cruz e Marques (2012), cada unidade de produto faz com que o insumo possa ser mais bem aproveitado. Denomina-se de economias de escala, pela qual se verifica a redução dos custos em resultado do maior número de habitantes. É expectável que os governos locais que apresentem maior número de habitantes consigam diminuir os custos. No modelo, enquadrou-se essa variável como de tipo nominal ou categórica. Justifica-se tal opção pela disparidade da população residente nos governos locais incluídos na amostra - sem essa transformação não seria possível garantir a normalidade da distribuição dos resíduos (Cunha, 2004; Henrichs \& Meza, 2017).

$\chi A ́ R E A i t$. A área, medida em $\mathrm{km}^{2}$, é outra variável do modelo. Justifica-se a inclusão pelas implicações decorrentes da área em razão dos custos que lhe estão associados. Por exemplo, governos locais que prestam serviços a uma área de maiores dimensões incorrem em maiores custos operacionais (Cruz \& Marques, 2012). Um dos casos poderá ser verificado pelo aumento do consumo de combustível, o qual elevará os custos para a prestação do serviço. De acordo com a anterior variável de controle, também a área foi classificada como variável de tipo nominal ou categórica (Cunha, 2004; Lima \& Silveira, 2018).

\subsection{Tratamento de dados e ferramentas estatísticas utilizadas}

Decorrente do acima exposto, preocupação maior existiu para que a normalidade da distribuição dos resíduos fosse assegurada às variáveis contínuas, que no modelo são as dependentes: custos dos consórcios, custos dos governos locais com os consórcios e custos totais. Num primeiro momento,

REvista de Administração Pública | Rio de Janeiro 54(5):1239-1259, set. - out. 2020 
verificou-se que a normalidade não foi cumprida. Face a essa necessidade, essas variáveis foram estimadas pela distribuição da proporção cumulativa por meio da fórmula de Blom (1959). Segundo o autor, a estimação é realizada pela fórmula $(r-3 / 8) /(w+1 / 4)$, correspondendo ao somatório do peso dos casos que originam o cálculo do ranking. Pela aplicação da fórmula, garantiu-se a normalidade na distribuição dos resíduos, a qual foi testada pelo teste de Shapiro-Wilk, em que se torna necessário que $p>.05$, o que foi verificado.

Em seguida, fez-se uso da ferramenta de análise univariada de variância com efeitos fixos. Tal ferramenta é usada quando constatadas duas condições: quando a coleta de dados é realizada em vários momentos e quando se pretende relacionar as variáveis do modelo entre si, ou seja, quando se pretende relacionar estatisticamente as variáveis independentes e contextuais. Ademais, essa técnica foi utilizada pela impossibilidade de garantir a homogeneidade de variância quando inclusas todas as variáveis dependentes num mesmo modelo.

A análise univariada inclui a regressão por quadrados mínimos ponderados, sendo o governo local a variável de ponderação. Três modelos foram executados, em que os custos dos consórcios, os custos dos governos locais com os consórcios e os custos totais surgem como variáveis dependentes. Para os cruzamentos, o teste de Levene foi utilizado. Esse teste é apropriado para verificar se a hipótese nula de que a variância de erro entre as variáveis dependentes são iguais entre os grupos. Tal hipótese foi confirmada $\operatorname{com} p=.277$, para o primeiro modelo; $\operatorname{com} p=.403$, para o segundo; e $p=.090$, para o terceiro. Os resultados são, portanto, críveis do ponto de vista estatístico.

Na sequência, analisa-se o teste de efeitos entre sujeitos para conhecer se as variáveis independentes e de controle têm efeito nas variáveis dependentes. Com esse cruzamento, tem-se a perceção de quais variáveis se relacionam estatisticamente. Depois, apresentam-se os resultados de estimativas de parâmetro com erro padrão robusto - com um intervalo de confiança de 95\%. As estimativas representam o sentido, positivo ou negativo, das variáveis estatisticamente relacionadas. Para a estatística, fez-se uso do Statistical Package for the Social Sciences (SPSS), versão 25.

\section{RESULTADOS E DISCUSSÃO}

Pela análise do Gráfico 1, verifica-se que os custos do consórcio, em média, têm aumentado. Curiosamente, os anos de 2013 e 2014 são o momento em que os custos dos próprios consórcios foram mais baixos. Com a crise financeira de 2015, atingiu-se o máximo de despesa, que diminuiu em 2016, algo esperado. Já em 2017 os custos dos consórcios aumentaram aos níveis de 2015. Se analisados os custos dos governos locais, verifica-se que o ano de 2015 é aquele que exigiu maiores recursos por parte dos municípios. Depreende-se uma descida média desses custos em 2016, invertendo-se essa tendência com o aumento dos custos para os governos locais em 2017. Apesar disso, fica claro que o custo para os governos locais tem sido menor no período de 2016 e 2017, em comparação com 2015. Essa tendência poderá ser verificada nos custos totais, ou seja, aumentaram em 2015 e diminuíram em 2016. Em 2017, verifica-se novo aumento, contudo abaixo dos níveis de 2015. 


\section{GRÁFICO 1 EVOLUÇÃO DOS CUSTOS COM 0 CONSÓRCIO}

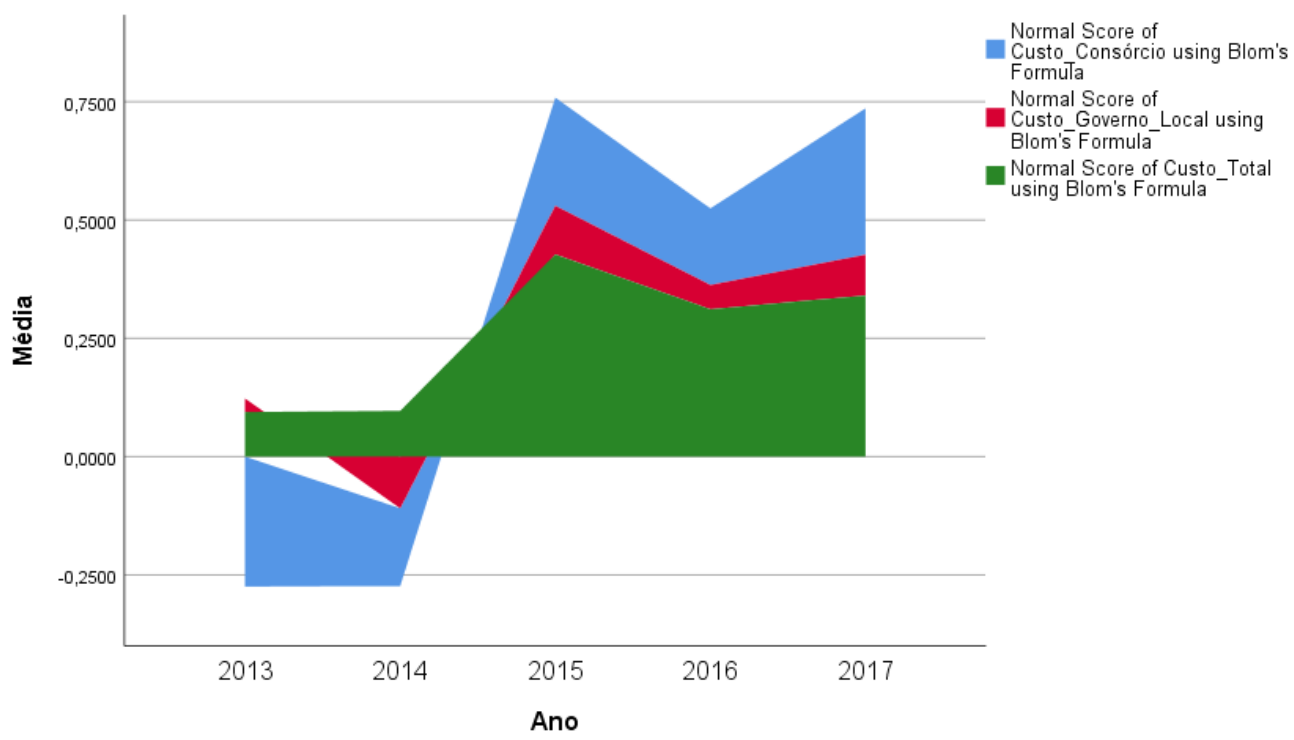

Fonte: Elaborado pelos autores com base nos dados coletados.

A curiosidade surge porque os custos médios totais foram os menores para todos os indicadores quando analisado o período de crescimento econômico. Desde que iniciada a crise, tais custos se elevaram, quando seria expectável que os governos locais reduzissem os repasses para todos os serviços, incluindo o dos resíduos sólidos. Essa realidade pode ser justificada pelos investimentos previstos pelos consórcios e que tiveram acolhimento quando houve o acordo de cooperação entre as unidades cooperantes.

Pela análise da Tabela 3, primeiro modelo, verifica-se que as variáveis consórcio $(p=.000)$, área $(p=.043)$ e ano $(p=.018)$ têm efeito no custo do consórcio. Entre a comparação dos consórcios, Cigres apresenta um custo médio menor ( $M=\mathrm{R} \$-.4352)$, numa comparação com Cirsures $(M=\mathrm{R} \$$.9237) e Ecotre ( $M=\mathrm{R} \$ 1.6350)$. Se alinhado com a área, verifica-se que os governos locais que servem áreas de até $157 \mathrm{~km}^{2}$ apresentam menores custos $(M=\mathrm{R} \$ 355.222,59)$ em relação com aquelas unidades que servem áreas maiores $(M=\mathrm{R} \$ 923.494,17)$. Pode-se concluir, portanto, que os consórcios que prestam serviços e que têm áreas de menor dimensão são aqueles que melhor potenciam a diminuição dos custos. Confirma-se, portanto, os resultados do estudo realizados por Nauro (2003), que reporta que os pequenos municípios são aqueles que mais se beneficiam da cooperação consorciada. Soma-se as explicações de Calderan (2013), nomeadamente de que a cooperação melhora a capacidade administrativa, entre outras, das estruturas cooperantes com vistas ao atingimento do melhor aproveitamento desses recursos. Em suma, esse resultado vai de encontro à literatura especializada, porquanto a sinergia criada pela cooperação pública potencia um melhor aproveitamento dos recursos e, consequentemente, um melhor desempenho operacional (Bel et al., 2010; Hefetz \& Warner, 2011). 


\section{TABELA 3 TESTE DE EFEITOS ENTRE SUJEITOS PELA REGRESSÃO POR QUADRADOS MÍNIMOS} PONDERADOS

\begin{tabular}{lccc} 
& Custo consórcio** & Custo governo local** & Custo total $^{* *}$ \\
\hline Intercepto & $.000^{*}(80.868)$ & $.000^{*}(14.675)$ & $.000^{*}(74.304)$ \\
Consórcio & $.000^{*}(14.797)$ & $.000^{*}(55.078)$ & $.000^{*}(13.986)$ \\
Ano & $.018^{*}(5.708)$ & $.936(.006)$ & $.562(.338)$ \\
População & $.115(2.506)$ & $.002^{*}(10.550)$ & $.606(.268)$ \\
Área & $.043^{*}(4.157)$ & $.008^{*}(7.323)$ & $.004^{*}(8.704)$ \\
Consórcio*Ano & $.504(.689)$ & $.620(.481)$ & $.923(.081)$ \\
Consórcio*População & $.531(.395)$ & - & $.102(2.702)$ \\
Consórcio*Área & $.236(1.414)$ & $.061(3.594)$ & $.952(.004)$ \\
Ano*População & $.457(.555)$ & $.405(.698)$ & $.782(.077)$ \\
Ano*Área & $.245(1.363)$ & $.891(.019)$ & $.691(.159)$ \\
Consórcio*Ano*População & $.509(437)$ & - & $.707(.141)$ \\
Consórcio*Ano*Área & $.519(.417)$ & $.369(814)$ & $.679(.172)$ \\
R-Quadrado & .678 & .745 & .678 \\
Graus de Liberdade & 13 & 11 & 13 \\
Estatística-F & $24.938^{*}$ & $27.060^{*}$ & $26.921^{*}$ \\
\hline
\end{tabular}

${ }^{*} \operatorname{sig} p<.05,{ }^{* *}$ ponderados por município

Estatística- $F$ entre parênteses

Fonte: Elaborada pelos autores com base nos dados coletados.

Pela análise do segundo modelo, são as variáveis consórcio $(p=.000)$, população $(p=.002)$ e área $(p=.008)$ que têm efeito nos custos para os governos locais. Mais uma vez, é o consórcio Cigres $(M=\mathrm{R} \$$-.4401) aquele que apresenta o menor custo médio. Seguem-se os consórcios Cirsures $(M=\mathrm{R} \$ .7238)$ e Ecotre $(M=\mathrm{R} \$ 1.5854)$. Os custos médios para os municípios que integram o consórcio Cirsures diminuíram de 2013 ( $M=\mathrm{R} \$ 1.346 .073,84)$ para $2017(M=\mathrm{R} \$ 1.098593,43)$. Em evolução inversa, os custos para os governos locais integrados nos consórcios Cigres e Ecotre aumentaram (de $M=\mathrm{R} \$ 185.226,75$ para $M=\mathrm{R} \$ 316.435,01$ e de $M=\mathrm{R} \$ 12.788 .041,59$ para $M=\mathrm{R} \$ 17.871 .816,70$, respectivamente). Em razão dos contratos assinados entre os municípios e consórcios, espera-se que os custos de financiamento aumentem. Assim, os resultados deste estudo dão vazão aos argumentos de Allers e Greef (2018), assim como a Frère, Leprince, e Paty (2014), ou seja, pode não se verificar quaisquer efeitos na diminuição das despesas/investimentos dos governos locais quando em cooperação. Porém, o custo para o município deverá ser balanceado com a quantidade e a qualidade do serviço.

Se considerada a variável população, municípios com mais de 20 mil habitantes apresentam maiores custos médios $(M=\mathrm{R} \$ 11.778 .677,74)$. Já os de menor dimensão, com até 20 mil habitantes, exibem custos médios menores $(M=\mathrm{R} \$ 463.815,68)$. A tendência se mantém quando considerada a área - aquelas com até $157,1 \mathrm{~km}^{2}$ apresentam menores custos médios $(M=\mathrm{R} \$ 336.006,02)$ do que aquelas de maior dimensão $(M=\mathrm{R} \$ 4.488 .894,68)$. Confirmam-se novamente as considerações de Cunha (2004), para 
quem a cooperação entre governos locais leva ao melhor aproveitamento das economias de escala. Pelo melhor aproveitamento das economias de escala, os custos para os governos locais diminuem.

No terceiro modelo, em que os custos totais surgem como variável dependente, são as variáveis consórcio $(p=.000)$ e área $(p=.004)$ que apresentam efeito estatístico significativo. Em razão da dimensão das unidades que o compõem, o consórcio Cigres apresenta os menores custos $(M=\mathrm{R} \$$-.4190), seguindo-se o Cirsures $(M=\mathrm{R} \$ .7785)$ e o Ecotre $(M=\mathrm{R} \$ 1.8149)$. Pela área, os resultados são em tudo semelhantes aos anteriores. Assim, áreas de menor dimensão não exigem os mesmos recursos financeiros do que as de maior dimensão ( $M=\mathrm{R} \$ 538574,26$ e $M=\mathrm{R} \$ 3786583,23$, respectivamente).

Numa súmula aos resultados dos testes de efeitos entre sujeitos, a variável consórcio e área servida provam ser aquelas que têm efeito nos gastos com os consórcios, para os governos locais e dos custos totais. Curiosamente, não existe qualquer relação de efeito quando consideradas as variáveis em conjunto. Apenas por uma vez a variável população mostra ter efeito nos custos, quando considerados os custos para os municípios como variável dependente. No seu conjunto, os resultados são expressivos em razão do elevado nível de explicação, medido pelo $R^{2}$ e em volta dos 70\%.

Num segundo momento, e pela estimativa de parâmetros com erro padrão robustos (ver Tabela 4), mostra-se expectável uma diminuição dos custos médios para os consórcios Cigres e Cirsures. Ao fim, os custos totais dos serviços de resíduos sólidos tendem a diminuir em ambos os casos. Para o financiamento do Cigres, é para os municípios com até 20 mil habitantes que se verifica a probabilidade estatística de redução mais provável dos custos médios de financiamento ( $M=\mathrm{R} \$-3.222)$.

\section{TABELA 4 RESUMO DA ESTIMATIVA DE PARÂMETRO COM ERRO PADRÃO ROBUSTO}

\begin{tabular}{|c|c|c|c|}
\hline & Custo consórcio & Custo governo local & Custo total \\
\hline Intercepto & $.000 *(12.837)$ & $.000 *(9.029)$ & $.000 *(16.901)$ \\
\hline Consórcio 1 & $.000^{\star}(-0.966)$ & $.000^{\star}(-16.493)$ & $.000 *(-12.780)$ \\
\hline Consórcio 2 & $.000^{\star}(-3.710)$ & $.000 *(-7.894)$ & $.000^{\star}(-7.011)$ \\
\hline Ano 1 & $.001^{*}(-3.348)$ & $.736(-.338)$ & $.995(.006)$ \\
\hline População 1 & $.002^{*}(-3.222)$ & $.000^{\star}(6.768)$ & $.356(.925)$ \\
\hline Área 1 & $.617(.501)$ & $.976(-.030)$ & $.285(-1.073)$ \\
\hline Consórcio 1Ano 1 & $.065(1.860)$ & $.464(.735)$ & $.583(-.550)$ \\
\hline Consórcio 2Ano 1 & $.149(1.452)$ & $.188(1.324)$ & $.323(-.992)$ \\
\hline Consórcio 1*População 1 & $.979(.026)$ & - & $.048^{\star}(-1.994)$ \\
\hline Consórcio 1*Área 1 & $.231(-1.202)$ & $.034^{\star}(-2.155)$ & $.766(-.298)$ \\
\hline Ano 1*População 1 & $.008^{\star}(2.686)$ & $.260(-1.133)$ & $.023^{\star}(2.299)$ \\
\hline Ano 1*Área 1 & $.143(-1.474)$ & $.421(-.807)$ & $.467(-.729)$ \\
\hline Consórcio 1*Ano 1*População 1 & - & - & $.341(-.954)$ \\
\hline Consórcio 1*Ano 1*Área 1 & - & $.276(1.096)$ & $.670(.427)$ \\
\hline
\end{tabular}

${ }^{*} \operatorname{sig} p<.05$ Estatística entre parênteses

Fonte: Elaborada pelos autores com base nos dados coletados. 
Pode-se concluir, portanto, que a existência dos consórcios potencializa a diminuição, de fato, dos custos de prestação de serviços de resíduos sólidos. Por essa razão, comprova-se que as redes ou networks se mostram um forte e potencial arranjo para os municípios, tendo em vista a redução dos preços de prestação de serviços (Bel \& Warner, 2015).

\section{CONCLUSÃO}

A cooperação intermunicipal se dá pelo incentivo dos municípios para a redução dos custos dos serviços que prestam (Feiock, 2007; Leroux \& Carr, 2007). Essa cooperação é propensa quando se trata de unidades do mesmo setor com limites geográficos próximos e que consentem a mesma necessidade de prestação de serviços (Feiock, 2007; Lamothe \& Lamothe, 2011). Admite-se que a cooperação tem efeito na diminuição das despesas para os governos locais, embora se exijam estudos vários para sua comprovação (Bel \& Warner, 2015).

Propôs-se neste estudo a descrição e a análise da relação entre a cooperação intermunicipal, por meio dos consórcios, com os gastos da cooperação pública e de financiamento. Selecionou-se o setor dos resíduos sólidos no Brasil, notadamente o estudo de três consórcios no período compreendido entre 2013 e 2017. Pela análise de efeitos, conclui-se que os consórcios e a área servida (medida em $\mathrm{km}^{2}$ ) têm efeito nos custos da cooperação pública. Para os governos locais, espera-se que a participação nos consórcios Cires e Cirsures represente uma diminuição de custos. Assim, temos que, para além do consórcio, os fatores contextuais têm grande efeito nos custos da cooperação. Ademais, são os pequenos municípios que apresentam mais vantagens do engajamento de cooperação. Se atentarmos que cerca de $70 \%$ dos municípios brasileiros tem até 20 mil habitantes, os resultados deste estudo se mostram relevantes para uma possível melhoria da gestão pública nacional. Nesse particular, e como opção à municipalização dos serviços ou à participação das organizações do setor privado, os consórcios se assumem como uma real e potencial alternativa, tendo em vista a redução dos custos de serviços de resíduos sólidos. A redução de custos poderá servir de incentivo para a cooperação intermunicipal, cujo potencial ainda não se desenvolveu plenamente.

A despeito de os dados ora apresentados serem robustos, do ponto de vista estatístico, é necessário realizar mais estudos sobre os efeitos dos arranjos consorciados nos custos de prestação dos serviços públicos. No caso do setor dos resíduos sólidos no Brasil, tais exames deverão incluir um período de análise mais alargado, para maior robustez dos dados. Por outro lado, carece de um melhor entendimento as razões para a evolução desses custos, nomeadamente quando, perante uma situação econômica e financeira favoráveis, se tenha verificado que os custos foram os menores. Por fim, o estudo sobre a cooperação intermunicipal deverá ser alargada a outros setores, para que se perceba se os resultados são comuns a todos eles. Decorre essa necessidade pela apresentação de características específicas de cada um desses setores, para além dos contextuais, os quais poderão ter efeito nos custos da prestação de serviços. 


\section{REFERÊNCIAS}

Abrucio, F. L. (1997). O impacto do modelo gerencial na administração pública: um breve estudo sobre a experiência internacional recente (Cadernos ENAP, n. 10). Brasília, DF: Escola Nacional de Administração.

Abrucio, F. L., Filippim, E. S., \& Dieguez, R. C. (2013). Inovação na cooperação intermunicipal no Brasil: a experiência da Federação Catarinense de Municípios (Fecam) na construção de consórcios públicos. Revista de Administração Pública, 47(6), 1543-1568.

Abrucio, F., Sano, H., \& Sydow, C. (2010). Radiografia do associativismo territorial brasileiro: tendências, desafios e impactos sobre as regiões metropolitanas. Regiões metropolitanas no Brasil (pp. 197-234). Washington, DC: BID.

Agranoff, R. (2014). Local governments in multilevel systems: Emergent public administration challenges. The American Review of Public Administration, 44(4), supl., 47S-62S.

Allers, M. A., \& De Greef, J. A. (2018). Intermunicipal cooperation, public spending and service levels. Local Government Studies, 44(1), 127-150.

Bel, G., Dijkgraaf, E., Fageda, X., \& Gradus, R. (2010). Similar problems, different solutions: Comparing refuse collection in the Netherlands and Spain. Public Administration, 88(2), 479-495.

Bel, G., Fageda, X., \& Mur, M. (2012). Does cooperation reduce service delivery costs? Evidence from residential solid waste services. Journal of Public Administration Research and Theory, 24(1), 85-107.

Bel, G., Fageda, X., \& Warner, M. E. (2010). Is private production of public services cheaper than public production? A meta-regression analysis of solid waste and water services. Journal of Policy Analysis and Management, 29(3), 553-577.

Bel, G., Hebdon, R., \& Warner, M. E. (2018). Beyond privatisation and cost savings: alternatives for local government reform. Local Government Studies, $44(2), 173-182$.

Bel, G., \& Warner, M. E. (2015). Inter-Municipal Cooperation and Costs: Expectations and evidence. Public Administration, 93(1), 52-67.
Bevir, M., \& Rhodes, R. A. W. (2001). Decentering tradition: interpreting British government. Administration \& Society, 33(2), 107-132.

Blaikie, N. (2009). Designing social research (2nd. ed.). Cambridge, UK: Polity Press.

Blatter, J., \& Blume, T. (2008). In Search of Co-variance, Causal Mechanisms or Congruence? Towards a Plural Understanding of Case Studies. Swiss Political Science Review, 14(2), 315-356.

Blom, G. (1958). Statistical elements and transformed beta variables. New York, NY: Wiley.

Boeschen, L., Koss, M. P., Figueredo, A. J., \& Coan, J. A. (2001). Experiential avoidance and posttraumatic stress disorder: a cognitive mediational model of rape recovery. Journal of Aggression, Maltreatment \& Trauma, 4(2), 211-245.

Bryman, A. (2015). Social research methods. (5. ed.). Oxford, UK: Oxford University Press.

Caldas, E. de L., \& Moreira, I. (2013). Políticas de desenvolvimento territorial e intermunicipalidade no Brasil: complementaridades e tensões. Sustentabilidade em Debate, 4(2), 41-61.

Calderan, T. B. (2013). Consórcio público Intermunicipal de Gerenciamento de Resíduos Sólidos Domésticos: um estudo de caso (Dissertação de Mestrado). Universidade do Vale do Taquari, Lajeado, RS.

Christensen, T. (2012). Post-NPM and changing public governance. Meiji Journal of Political Science and Economics, 1(1), 1-11.

Cruz, M. D. C. M. T. (2001). Consórcios Intermunicipais de Saúde, Educação e Assistência Social no Estado de São Paulo. In CEPAM (Ed.), Consórcio: uma Forma de Cooperação Intermunicipal. São Paulo, SP: Autor.

Cruz, N. F., \& Marques, R. C. (2012). Mixed companies and local governance: no man can serve two masters. Public administration, 90(3), 737-758.

Cunha, R. E. (2004). Federalismo e relações intergovernamentais: os consórcios públicos como instrumento de cooperação federativa. Revista do Serviço Público, 55(3), 5-36.

Denhardt, R. B., \& Denhardt, J. V. (2000). The new public service: Serving rather than steering. Public Administration Review, 60(6), 549-559. 
Feiock, R. C. (2007). Rational choice and regional governance. Journal of Urban Affairs, 29(1), 47-63.

Ferreira, C. F. A., \& Jucá, J. F. T. (2017). Metodologia para avaliação dos consórcios de resíduos sólidos urbanos em Minas Gerais. Engenharia Sanitária e Ambiental, 22(3), 513-521.

Fonseca, F. (2013). Consórcios públicos e as agendas do Estado brasileiro (pp. 29-40). São Paulo, SP: Fundação Perseu Abramo.

Frère, Q., Leprince, M., \& Paty, S. (2014). The impact of intermunicipal cooperation on local public spending. Urban Studies, 51(8), 1741-1760.

Gerring, J. (2004). What is a case study and what is it good for? American Political Science Review, 98(2), 341-354.

Hefetz, A., \& Warner, M. E. (2011). Contracting or public delivery? The importance of service, market, and management characteristics. Journal of Public Administration Research and Theory, 22(2), 289-317.

Henrichs, J. A., \& Meza, M. L. F. G. (2017). Governança multinível para o desenvolvimento regional: um estudo de caso do Consórcio Intermunicipal da Fronteira. Revista Brasileira de Gestão Urbana, 9(1), 124-138.

Hood, C. (1991). A public management for all seasons? Public administration, 69(1), 3-19.

Hulst, J. R., \& Montfort, A. J. G. M. van (2012). Institutional features of inter-municipal cooperation: Cooperative arrangements and their national contexts. Public Policy and Administration, 27(2), 121-144.

Instituto Brasileiro de Geografia e Estatística. (2018). Perfil dos Municípios Brasileiros. Recuperado de https://ww2.ibge.gov.br/home/estatistica/economia/ perfilmunic/2015/default.shtm

Jooste, S. F., \& Scott, W. Richard. (2012). The publicprivate partnership enabling field: evidence from three cases. Administration \& Society, 44(2), 149-182.

Kelly, J. (2007). The curious absence of intermunicipal cooperation in England. Public Policy and Administration, 22(3), 319-334.

Klijn, E.-H. (2008). Complexity theory and public administration: what's new? Key concepts in complexity theory compared to their counterparts in public administration research. Public Management Review, 10(3), 299-317.

Klijn, E.-H., Koppenjan, J., \& Termeer, K. (1995). Managing networks in the public sector: a theoretical study of management strategies in policy networks. Public Administration, 73(3), 437-454.

Laczynski, P., \& Teixeira, M. A. C. (2012). Os limites de um consórcio intermunicipal em condições assimétricas de poder: o caso do Cinpra no Maranhão. Cadernos Adenauer, 12(4), 141-160.

Lamothe, M., \& Lamothe, S. (2011). To trust or not to trust? What matters in local government-vendor relationships? Journal of Public Administration Research and Theory, 22(4), 867-892.

Leroux, Kelly, \& Carr, J. B. (2007). Explaining local government cooperation on public works: Evidence from Michigan. Public Works Management \& Policy, 12(1), 344-358.

Lima, R. C. de A., \& Silveira, R. M. S., Neto. (2018). Secession of municipalities and economies of scale: evidence from Brazil. Journal of Regional Science, 58(1), 159-180.

Linhares, P. T. F. S., Messenberg, R. P., \& Ferreira, A. P. L. (2017). Transformações na federação brasileira: o consórcio intermunicipal no Brasil do início do século XXI. Boletim de Análise Político-Institucional, 12, 67-74.

Machado, J. A., \& Andrade, M. L. C. (2014). Cooperação intergovernamental, consórcios públicos e sistemas de distribuição de custos e benefícios. Revista de Administração Pública, 48(3), 695-720.

Massardier, G., \& Sabourin, E. (2013). Internationalization and dissemination of rural territorial development public policies: model hypotheses for Latin America. Sustentabilidade em Debate, 4(2), 83-100.

Matos, F., \& Dias, R. (2012). Cooperação intermunicipal na bacia do rio Paraopeba. Revista de Administração Pública, 46(5), 1225-1250.

Mello, D. L. (1997). Associativismo como instrumento de desenvolvimento dos governos locais: a experiência brasileira e de outros países. Revista de Administração Pública, 31(6), 55-66. 
Mello, L., \& Lago-Peñas, S. (2013). Local government cooperation for joint provision: the experiences of Brazil and Spain with inter-municipal consortia. In S. Lago-Peñas \& J. Martinez-Vazquez. (Ed.), The Challenge of Local Government Size (Chap. 9, pp. 221-241). Northampton, UK: Edward Elgar Publishing.

Nagashima, L. A., Barros, C., Jr., Andrade, C. C., Silva, E. T., \& Hoshika, C. (2011). Gestão integrada de resíduos sólidos urbanos-uma proposta para o município de Paranavaí, Estado do Paraná, Brasil. Acta Scientiarum Technology, 33(1), 39-47.

Niaounakis, T., \& Blank, J. (2017). Inter-municipal cooperation, economies of scale and cost efficiency: an application of stochastic frontier analysis to Dutch municipal tax departments. Local Government Studies, 43(4), 533-554.

Osborne, S. P. (2006). The New Public Governance? Public Management Review, 8(3), 377-387.

Osborne, S. P. (Ed.). (2010). The new public governance: Emerging perspectives on the theory and practice of public governance. London, UK: Routledge.

Oström, E. (1996). Crossing the great divide: coproduction, synergy, and development. World development, 24(6), 1073-1087.

O’toole, Laurence J., Jr., \& Meier, K. J. (2004). Public management in intergovernmental networks: Matching structural networks and managerial networking. Journal of public administration research and theory, 14(4), 469-494.

Peters, B. G. (2004). Governance and public bureaucracy: new forms of democracy or new forms of control? Asia Pacific Journal of Public Administration, 26(1), 3-15.

Pollitt, C. (1990). Managerialism and the public services: the Anglo-American experience. Oxford, UK: Blackwell Publishing.

Pollitt, C., \& Bouckaert, G. (2017).Public management reform: a comparative analysis-into the age of austerity. Oxford, UK: Oxford University Press.

Provan, K. G., \& Milward, H. B. (1999). Do networks really work? A Framework for Evaluating Publicsector Organizational Networks. Academy of
Management Proceedings. Recuperado de https:// doi.org/10.5465/apbpp.1999.27628083

Puey, E. P., Ferran, J. M., \& Mussons, C. P. (2018). Beyond size: overcoming fragmentation by intermunicipal associations in Spain? The case of Catalonia. International Review of Administrative Sciences, 84(4), 639-658.

Rhodes, R. A. W. (2000). Governance and public administration. Debating governance, 5490.

Rhodes, R. A. W. (2007). Understanding governance: ten years on. Organization Studies, 28(8), 1243-1264.

Sabourin, E. P., Massardier, G., \& Sotomayor, O. (2016). As políticas de desenvolvimento territorial rural na América latina: uma hibridação do referencial e da implementação. Mundos PluralesRevista Latinoamericana de Políticas y Acción Pública, 3(1), 75-98.

Santos, A. M dos, \& Giovanella, L. (2014). Governança regional: estratégias e disputas para gestão em saúde. Revista de Saúde Pública, 48(4), 622-631.

Shrestha, M. K., \& Feiock, R. C. (2011). Transaction cost, exchange embeddedness, and interlocal cooperation in local public goods supply. Political Research Quarterly, 64(3), 573-587.

Silvestre, H. C. (2019). A (Nova) Governança Pública. Brasília, DF: Coleção Gestão Pública ENAP/SBAP.

Silvestre, H. C. (2010). Gestão pública: modelos de prestação no serviço público. Lisboa, Portugal: Escolar Editora.

Silvestre, H. C., Marques, R. C., Dollery, B., \& Correia, A. M. (2019). Shared services in Brazilian local government: Urban development in small counties. Public Administration, 97(3), 686-702.

Silvestre, H. C., Marques, R. C., \& Gomes, R. C. (2018). Joined-up Government of utilities: a metareview on a public-public partnership and intermunicipal cooperation in the water and wastewater industries. Public Management Review, 20(4), 607-631.

Silveira, R. C. E., \& Philippi, L. S. (2008). Consórcios públicos: uma alternativa viável para a gestão regionalizada de resíduos sólidos urbanos. Redes, 13(1), 205- 224. 
Souza, R. G. D., \& Cordeiro, J. S. (2010). Mapeamento cognitivo e Balanced Scorecard na gestão estratégica de resíduos sólidos urbanos. Gestão \& Produção, 17(3), 483-496.

Spink, P. (2005). The inter-municipal consortia in Brazil: an institutional introduction. In Anales de $10^{\circ}$ Congresso Internacional del Clad, Santiago, Chile.

Stewart, J. (2012). Multiple-case study methods in governance-related research. Public Management Review, 14(1), 67-82.

Torfing, J., \& Sørensen, E. (2014). The European debate on governance networks: Towards a new and viable paradigm? Policy and Society, 33(4), 329-344.

Urban, R. C. (2016). Índice de adequação do gerenciamento de resíduos sólidos urbanos como ferramenta para o planejamento: aplicação no estado de São Paulo. Revista Engenharia Sanitária, 21(2), 367-377.

Williamson, O. E. (1999). Public and private bureaucracies: a transaction cost economics perspectives. The Journal of Law, Economics, and Organization, 15(1), 306-342.

Yin, R. K. (2015). Estudo de caso: planejamento e métodos. São Paulo, SP: Bookman.

Zafra-Gómez, J. L., Prior, D., Díaz, A. M. P., \& López-Hernández, A. M. (2013). Reducing costs in times of crises: delivery forms in small and medium sized local government waste management services. Public Administration, 91(1), 51-68.

\section{Dituzaya Panguila da Silva}

https://orcid.org/0000-0002-8683-8351

Mestrando em Políticas Públicas pelo Instituto Universitário de Lisboa-ISCTE.

E-mail: dpsaa2@iscte-iul.pt

\section{Hugo Consciência Silvestre}

https://orcid.org/0000-0001-7654-6577

Doutor em Ciências da Administração na especialidade de Gestão Pública; Professor Associado do Instituto de Ciências Sociais Aplicadas da Universidade da Integração Internacional da Lusofonia Afro-brasileira (UNILAB); Líder do Núcleo de Políticas \& Administração Pública (NPAP). E-mail. hmcsilvestre@unilab.edu.br

\section{Alfa Aliu Embalo}

https://orcid.org/0000-0002-4666-0309

Mestrando em Gestão Autárquica pelo Instituto Superior de Educação e Ciências de Lisboa-ISEC.

E-mail: alfaaliuembalo@hotmail.com 